\title{
Local and Global Fiber Tractography in Patients with Epilepsy
}

\author{
C. Anastasopoulos, M. Reisert, V.G. Kiselev, T. Nguyen-Thanh, A. Schulze-Bonhage, J. Zentner, and I. Mader
} O- $=$

\begin{abstract}
BACKGROUND AND PURPOSE: Fiber tractography is increasingly used in the preoperative evaluation of endangered fiber bundles. From a clinical point of view, an accurate and methodologically transparent procedure is desired. Our aim was to evaluate the recently described global tracking algorithm compared with other established methods, such as deterministic and probabilistic tractography.
\end{abstract}

MATERIALS AND METHODS: Twenty patients, candidates for excision of epileptogenic lesions, were subjected to higher-angular resolution diffusion imaging-based fiber tractography. Seed points were created without manual bias, predominantly by FreeSurfer and voxel-based atlases. We focused on 2 important fiber bundles, namely the descending motor pathways and the optic radiation. Postoperatively, the accuracy of the predicted fiber route was controlled by structural MR imaging and by inflicted functional deficits.

RESULTS: Among the 3 evaluated methods, global tracking was the only method capable of reconstructing the full extent of the descending motor pathways, including corticobulbar fibers from the area of face representation. Still, probabilistic tractography depicted the optic radiation better, especially the Meyer loop. The deterministic algorithm performed less adequately.

CONCLUSIONS: The probabilistic method seems to be the best balance between computational time and effectiveness and seems to be the best choice in most cases, particularly for the optic radiation. If, however, a detailed depiction of the fiber anatomy is intended and tract crossings are implicated, then the computationally time-consuming global tracking should be preferred.

ABBREVIATIONS: DMP = descending motor pathways; FA = fractional anisotropy; FACT = Fiber Assignment by Continuous Tractography; OR $=$ optic radiation; $\mathrm{SLF}=$ superior longitudinal fascicle

B efore neurosurgical operations, especially those demanding resection of lesions adjacent to functionally critical areas, the structural and functional anatomy of the region must be evalu-

Received February 21, 2013; accepted after revision April 24.

From the Clinic of Neuropediatrics and Neuromuscular Diseases (C.A.), Medical Physics, Department of Radiology (M.R., V.G.K.), Epilepsy Center (A.S.-B.), and De partments of Neurosurgery (J.Z.), and Neuroradiology (I.M.), University Medical Center Freiburg, Freiburg, Germany; and Department of Radiology (T.N.-T.), Hue University College of Medicine and Pharmacy, Hué, Vietnam.

T.N.T. and I.M. were supported by the Comprehensive Cancer Center Freiburg, Freiburg, Germany (Seeding Grant 1027013110). V.G.K. and I.M. were supported by the German Research Council (DFG: KI 1089/3-1, MA 2343/4-1).

Paper previously presented in part at: 8th Annual World Congress of IBMISPS on Brain, Spinal Cord Mapping and Image Guided Therapy, June 8-10, 2011; San Francisco, California.

Please address correspondence to Constantin Anastasopoulos, Clinic of Neuropediatrics and Neuromuscular Diseases, Pediatric Hospital, University Medical

Center Freiburg, Mathildenstr. 1, 79106 Freiburg, Germany; e-mail: constantin.anastasopoulos@uniklinik-freiburg.de

-- Indicates open access to non-subscribers at www.ajnr.org

EIndicates article with supplemental on-line table.

4 Indicates article with supplemental on-line figures.

http://dx.doi.org/10.3174/ajnr.A3752 ated. In epilepsy neurosurgery, where protection of "eloquent" brain areas is essential, knowledge of the fiber course in altered anatomic conditions is beneficial. Therefore, diffusion tractography has been increasingly used for neurosurgical planning. ${ }^{1}$

Tractography algorithms use the information that is obtained by multiple-gradient DWI on the basis of the anisotropic character of motion of free water molecules along myelinated fibers. ${ }^{2}$ Various tractography algorithms are available, with 2 main categories being local (further subdivided into deterministic and probabilistic) and global methods (On-Line Fig 1). The deterministic fiber assignment by continuous tractography (FACT) algorithm is increasingly used for preoperative and intraoperative tractography. ${ }^{3,4}$ Most deterministic algorithms follow the principal orientation of diffusion. This approach leads to erroneous results, if there are fibers within a voxel running in different directions. ${ }^{5}$ In addition, fibers with a strong curvature may be difficult to reconstruct. For example, the extent of the optic radiation (OR) of the Meyer loop is frequently underestimated. ${ }^{6}$ Probabilistic tractography assesses the probability that a voxel is connected to a given start point, by means of iterative random walks. ${ }^{7}$ This method exploits the statistical nature of the information ob- 
tained by DWI and determines the most probable mathematic pathway. ${ }^{8}$ In contrast, global methods process the entire diffusion information simultaneously and generate a whole-brain reconstruction. Global tracking simulates an annealing procedure where-during a "decrease of temperature"-line elements of subvoxel size, representing the diffusion anisotropy, bind together. Finally, only aligned elements remain bound to each other according to the diffusion information of the brain. ${ }^{9}$ It has been suggested that global tractography overcomes the common issue of fiber crossings. ${ }^{10}$ The superiority of this algorithm has been proven in a study comparing 10 different algorithms on a phantom that mimics fiber crossing and bending. ${ }^{11}$ The main disadvantage of global methods is the long calculation time, whereas the disadvantage of local methods, particularly the FACT algorithm, is the accumulation of errors during the algorithm propagation. $^{5}$

Newer tractography algorithms outrank certain features of older algorithms. For example, probabilistic tractography is more robust than deterministic tractography in areas of high uncertainty (ie, areas of high signal noise or fiber crossings). ${ }^{12}$ To date, it is not clear which particular tractographic approach has the appropriate features for certain anatomic or clinical questions. Therefore, it is essential to perform comparisons, not only by means of phantoms, ${ }^{11}$ but also in an appropriate clinical setting. This study aimed at evaluating the clinical usefulness of global tractography as a preoperative tool and compared its performance with the widely used FACT and a probabilistic algorithm. The focus was on the depiction of the major fiber structures' descending motor pathways (DMP) or OR.

\section{MATERIALS AND METHODS \\ Patients}

A total of 20 prospectively recruited consecutive patients (mean age, 27 years; age range, 3-66 years; 11 women) with drug-resistant epilepsy due to various supratentorial lesions were investigated. The inclusion criteria were 1) the presence of a benignappearing lesion, adjacent to either the corticospinal tract or the OR; and 2) intended total lesion resection or minor loss of function postoperatively. The exclusion criterion was a malignant appearance (eg, major space-occupying lesion) with perifocal edema. Seven patients presented with focal cortical dysplasia, 5 with cavernoma, 3 with intra-axial low-grade tumors, 2 with hippocampal sclerosis, 1 with perinatal ischemic stroke, 1 with schizencephaly, and 1 with an arteriovenous malformation (On-line Table). The treatment procedure in each patient was individually determined in consensus decision-making sessions among neurologists, neuroradiologists, and neurosurgeons in the Neurocenter of the University Medical Center (Freiburg, Germany). All available information, including tractography results, was taken into account. The interval between preoperative evaluation and operation ranged from 3-19 days. The rationale behind tractography in the preoperative planning was the concern about the extent of the intended resection and subsequent functional outcome. The tractography analysis was performed by 2 neuroradiologists in training (C.A. and T.N.T.) under supervision of a senior neuroradiologist (I.M.). Preoperative and postoperative visual fields were assessed by Goldmann perimetry. The postop- erative seizure outcome was assessed from 6-17 months after surgery (mean, 10 months) and was categorized according to the Engel and the International League Against Epilepsy classifications. ${ }^{13,14}$ The procedures were approved by the local ethics committee.

\section{Image Acquisition}

All measurements were performed by means of a 3T scanner (Magnetom Trio; Siemens, Erlangen, Germany). For DWI, a single-shot spin-echo-planar imaging sequence by use of a 12-channel head coil was applied (TR, $10.5 \mathrm{~s}$; TE, $96 \mathrm{~ms}$; resolution, $2^{3}$ $\mathrm{mm}^{3} ; 61$ diffusion-encoding directions; effective b-value, 1000 $\mathrm{s} / \mathrm{mm}^{2}$ ). The orientation distribution function in each voxel was extracted from higher-angular resolution diffusion imaging. ${ }^{15}$ In all patients, a T1WI magnetization prepared rapid acquisition of gradient-echo sequence with a $1-\mathrm{mm}^{3}$ resolution was obtained (TR, $2.2 \mathrm{~s}$; TE, $2.15 \mathrm{~ms}$; TI, $1.1 \mathrm{~ms}$ ) preoperatively and postoperatively. Preoperative structural images were coregistered with the DWI and were subsequently segmented into white matter, gray matter, and CSF by use of Statistical Parametric Mapping 8 (Wellcome Department of Imaging Neuroscience, London, UK), running under Matlab2009b (MathWorks, Natick, Massachusetts). A brain mask was created, restricting the algorithms' calculations to the white matter and the adjacent 2 voxels of gray matter volume.

\section{Seed Point Selection}

A 2-seed point procedure was used for fiber tractography. For each of the 2 fiber structures, 2 ROIs served as end points: 1 cortical and 1 either in the ipsilateral brain stem for the DMP or the thalamus for the OR. In patients with obvious anatomic deviations (patients 3 and 9), motor cortex seed points were derived from fMRI. For fMRI, contiguous multisection echo-planar imaging was used (TR, $2.61 \mathrm{~s}$; TE, $30 \mathrm{~ms}, 3^{3} \mathrm{~mm}^{3}$, active fist-clenching and tongue movement vs rest, respectively, $1 \mathrm{~Hz}, 9$ measurements). After preprocessing, the peak maxima of the $t$-values of the statistical maps $\left(P_{\text {corr }(\mathrm{FWE})}<.05\right)$ were enlarged by $3 \mathrm{~mm}$ in every direction to extend into white matter and were taken as seed points for the primary motor area.

In all other patients, cortical seed points were obtained by a standard automated parcellation with the FreeSurfer (http:// surfer.nmr.mgh.harvard.edu/) algorithm. ${ }^{16}$ Brain stem/thalamic seed points were derived from a voxel-based atlas. ${ }^{17}$ The ROIs were registered to the individual coordinate system of the patients' T1WI and DWI datasets. The precentral gyrus served as the cortical origin and the ipsilateral cerebral crus ROI as the second end point of the DMP in patients \#1-10; the pericalcarine cortex served as the cortical end point for the OR and the lateral geniculate nucleus as the second end point for the OR in patients \#1120. Although the ROIs were atlas derived, some minor corrections were made: 1) the size of the brain stem ROI was halved to the ipsilateral cerebral peduncle; and 2) the lateral geniculate nucleus ROI was enlarged in every direction up to $4 \mathrm{~mm}$, correcting its relatively small atlas size to match neuroanatomic studies. ${ }^{18}$

\section{Fiber Tractography}

We made no manual interference when we applied the tractography methods. All 3 tractography methods were successively used 

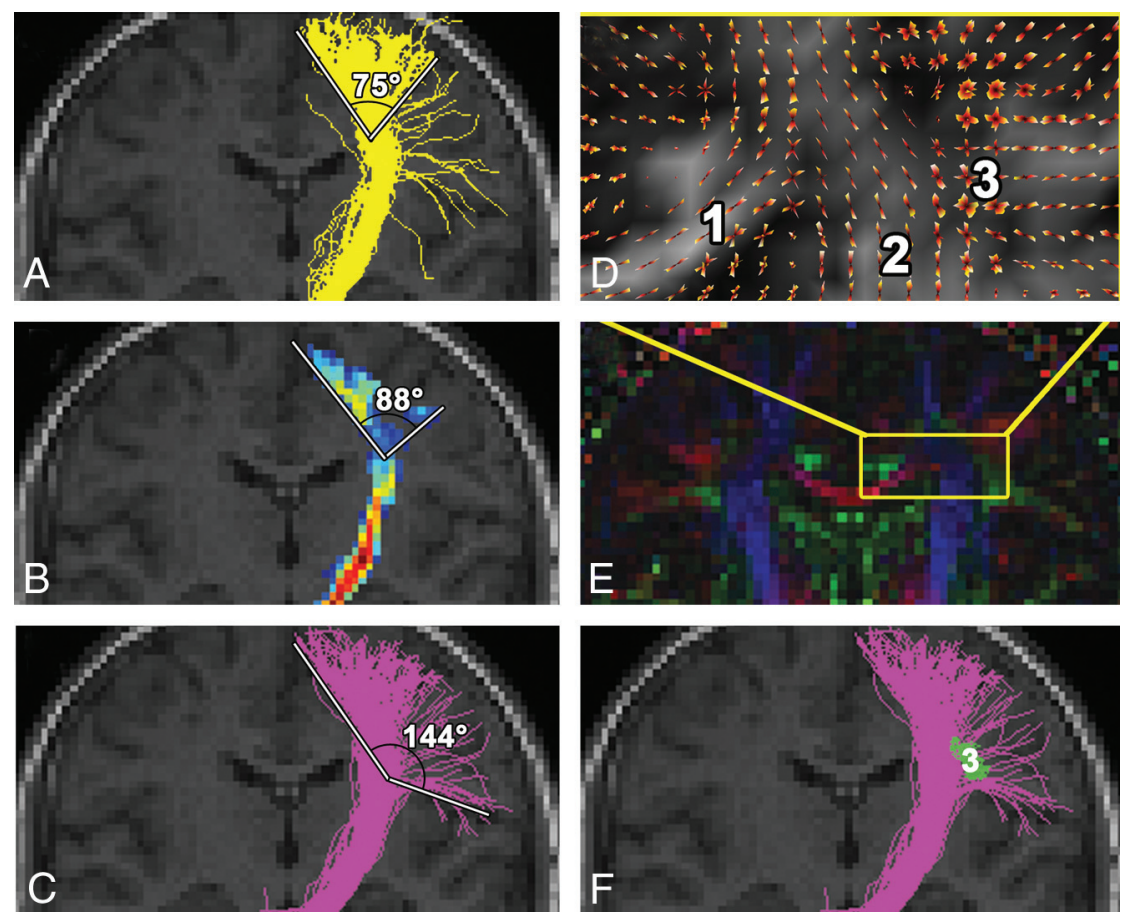

FIG 1. Depiction of the left descending motor pathways on the healthy side in patient 3 and the fan angle measurement performed. FACT $(A)$, probabilistic $(B)$, and global ( $C$ and $F$ ) tractography show approximately the same route and extent along the internal capsule. Note differences in the reconstruction of fibers arising from the lateral area of face cortical representation. These corticobulbar fibers are best depicted by global tracking, as this method is least susceptible to fiber crossings in the semiovale center $(D)$ (orientation distribution function of the diffusion signal) and (E) (principal direction of the diffusion tensor shown as color-coded map) between the corpus callosum (7), corona radiata (2), and SLF (3). FACT and probabilistic tractography cannot overcome the crossing with the SLF (3, green in $F$, oriented perpendicular to the plane of the figure), with the results shown in On-Line Fig 2 in mind.

under the same conditions: 1) all algorithms were applied on the same diffusion data; and 2) the brain mask and 3) the pair of ROIs used were the same. The computation time span on a standard 64-bit platform was 30 minutes for FACT, 3 hours for probabilistic maps of connectivity, and 20 hours for global tracking. The results of tractography in the preoperative planning were analyzed by the same in-house visualization tool (http://www. uniklinik-freiburg.de/mr/live/arbeitsgruppen/diffusion/fibertools_ en.html) and were superimposed on preoperative and, in a second step, postoperative structural imaging. For comparison of the tractography results for the DMP, the fan angle from the fibers arising in the foot area to the fibers from the face area was measured (Fig 1). The results of the different tractography methods for the OR were compared based on the presence of the Meyer loop and its distance to the tip of the temporal pole.

For FACT, particular stopping criteria were fractional anisotropy $(\mathrm{FA})<0.1$ and a curvature between 2 consecutive steps $\geq 90^{\circ}$. The liberal curvature was chosen to allow the detection of strong fiber bending.

In our current study, an extension to the probabilistic index of connectivity method ${ }^{7}$ was adopted, which calculates the probability that a voxel is connected to the selected ROI by performing random walk iterations and counting the visits. At the same time, the directional information of the curves passing through the voxel is preserved. The probability information of voxels receiving visits from opposing directions creates probabilistic maps of connectivity. ${ }^{8}$ It allows the depiction of the mathematically most probable pathway between 2 ROIs (On-Line Fig 1). Parameters were $10^{5}$ random walk iterations from every seed region voxel and applying an exponent of 4 to the eigenvalues, whereas the stopping criterion was FA $<0.1$.

Global fiber tractography methods reconstruct all brain fibers simultaneously by finding a configuration that best describes the diffusion data., ${ }^{9,19}$ In global tracking, the reconstructed fibers are built by small line segments (initially hundreds in each voxel) that represent the diffusion anisotropy (On-Line Fig 1E-G). During optimization, an iterative process is repeated with an order of $10^{8}$ iterations, and the elements bind together in an annealing simulation. This behavior is governed by an interaction between the line elements and the measured data. A detailed description of global tracking is given by Reisert et al. ${ }^{10}$

\section{RESULTS \\ Fiber Tractography in \\ Presurgical Planning}

In patients 1-10, preoperative reconstruction of the DMP by FACT and probabilistic algorithm, in both healthy and pathologic hemispheres, involved fibers arising from the precentral gyrus. Predominantly fibers from the arm and foot cortical representation, through the internal capsule and to the cerebral peduncle, were reconstructed. The mean fan angle for FACT was $92^{\circ}$ (range, 66$114^{\circ}$ ) and for the probabilistic algorithm, $97^{\circ}$ (range, 50-122 ; Fig 1). In contrast, the extent of the DMP pathways achieved by global tracking was broader in all patients, with multiple fibers starting from the foot, trunk, arm, and face areas of the precentral gyrus, achieving a mean fan angle of $138^{\circ}$ (range, $118-156^{\circ}$; Fig 1). This algorithm was thus capable of depicting corticospinal, as well as corticobulbar, pathways.

In patients 11-20, the FACT algorithm depicted the stem of the OR running from the lateral geniculate nucleus caudally toward the ipsilateral pericalcarine cortex. The Meyer loop could be partially depicted in 3 of 10 patients (mean distance to the tip of the temporal pole, $41 \mathrm{~mm}$; range, $39-43 \mathrm{~mm}$ ). The probabilistic maps of connectivity depicted the OR in all 10 patients, including the Meyer loop in 9 of 10 patients (mean distance to the tip of the temporal pole, $34 \mathrm{~mm}$; range, $23-40 \mathrm{~mm}$ ). Global tracking reconstructed only the main part of the OR and failed to reconstruct the Meyer loop in all 10 patients. In patient \#13, reconstruction of the Meyer loop was missing in all methods because of impaired image quality caused by motion artifacts.

\section{Postoperative Evaluation}

In 7 of 10 patients with tractography of the DMP and in 8 of 10 patients with tractography of the OR, an excision of the alleged or 

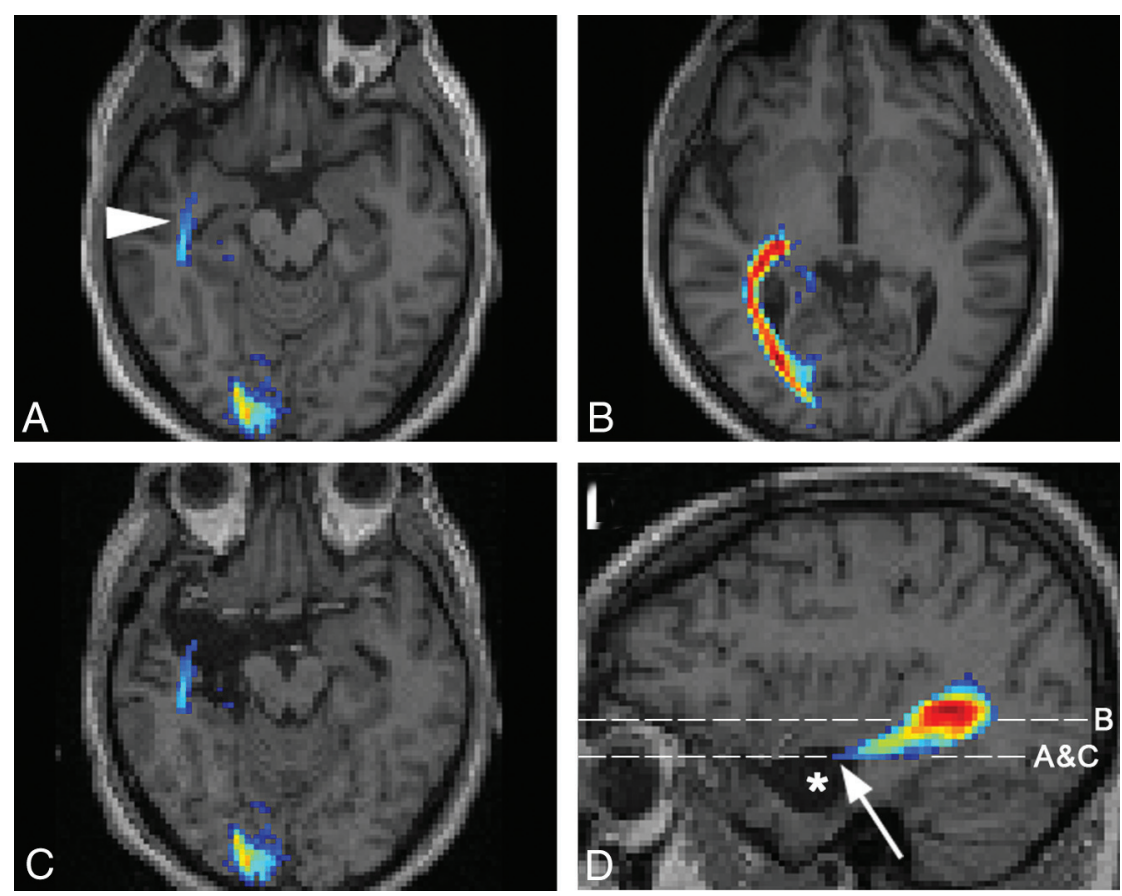

FIG 2. Assessment of the OR in patient 14 with a right hippocampal sclerosis. Results of probabilistic tractography $(A)$ for the right Meyer loop (arrowhead) and (B) for the main part of the $\mathrm{OR}$ are shown. The preoperative OR reconstruction by the probabilistic algorithm is superimposed onto postoperative images $(C$ and $D$, asterisk $=$ resection area, arrow $=$ anterior extent of Meyer (oop). Postoperatively, the patient presented with a partial visual field defect in the left upper quadrant.
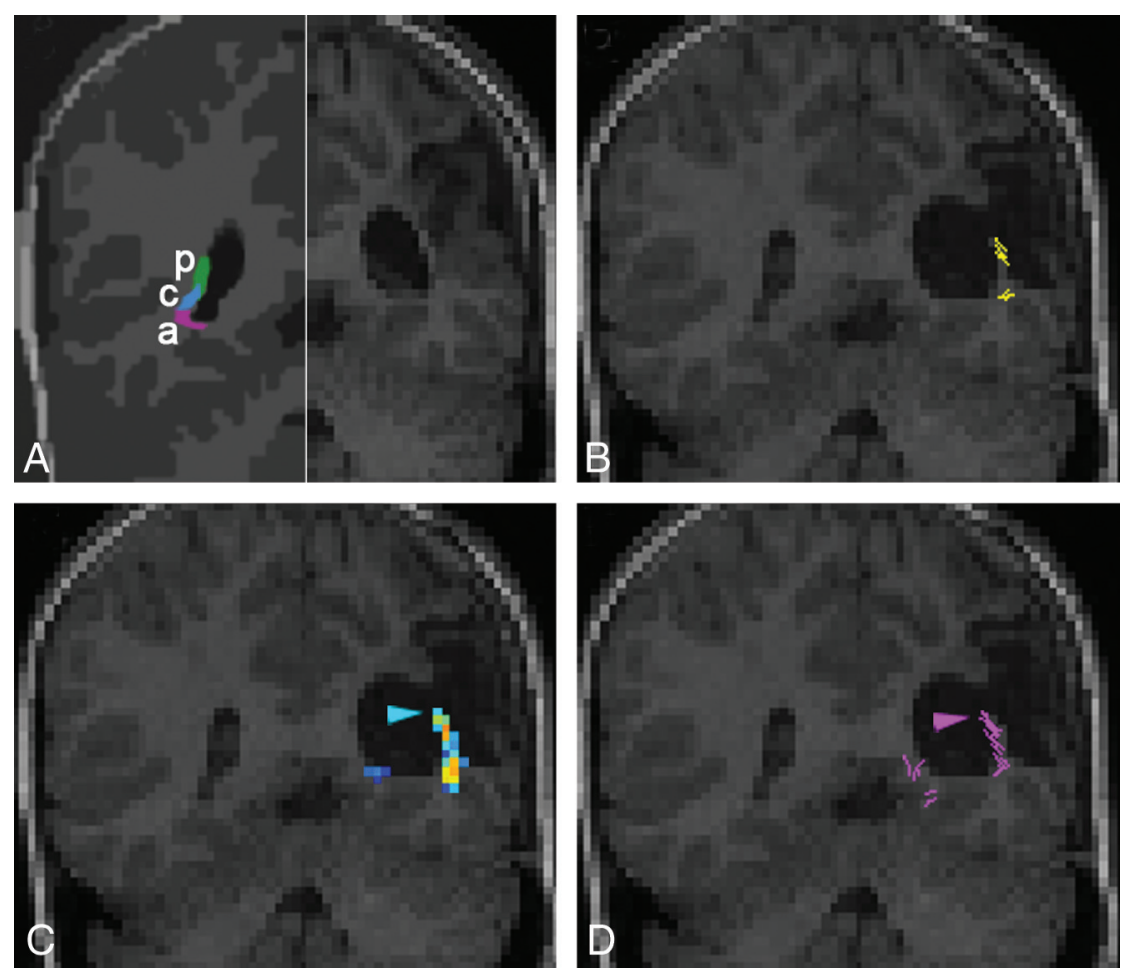

FIG 3. Coronal sections through the $\mathrm{OR}$ at the level of the trigone of the lateral ventricle in patient 15 with a perinatal stroke. Scheme drawing $(A)$ of the functional organization of the OR on the healthy side $\left(\right.$ redrawn $\left.{ }^{26}\right)$, indicating the course of the posterior ( $\left.\mathrm{p}\right)$, anterior (a), and central (c) bundles, carrying fibers of the lower quadrant, upper quadrant, and center of the contralateral visual field, respectively. Preoperative TWWI of the pathologic side (right half, $A$ ). Postoperatively, the patient had a lower right quadrant anopia. Preoperative tractography superimposed on the postoperative coronal $T(B-D)$. The arrowhead indicates that the posterior bundle of the OR was within the resection area. It is missed by FACT (B) but is depicted by probabilistic $(C)$ and global $(D)$ tractography. suspected epileptogenic focus was performed. During postoperative follow-up, 9 patients remained seizure-free; 5 patients had less frequent seizures than before surgery; and, in 1 patient, the seizure type and frequency remained unchanged. Surgical treatment was thought to be inappropriate in 5 patients ( 3 with tractography of the DMP and 2 of the OR), as a functional impairment may have ensued, because of the vicinity to the main part of the fiber pathways or to the respective cortex.

To re-evaluate our findings, we superimposed preoperative tractographic results on the postoperative imaging in patients having received surgery. In 2 patients, the detected fibers were presumably damaged during the operation. In patient 14, a right selective amygdalohippocampectomy was performed. Preoperatively, only the probabilistic algorithm successfully depicted the OR, including the Meyer loop (Fig 2). Postoperatively, the patient had a small left upper visual field defect. The preoperative probabilistic OR reconstruction was superimposed on the T1WI acquired 3 months postoperatively. The anterior extent of the reconstructed Meyer loop was now situated in the area that had been excised. In patient 15, a leftsided extended perisylvian lesionectomy of perinatal infarcted tissue was performed (Fig 3). Preoperatively, reconstruction of the OR was intended by the 3 algorithms. Probabilistic and global reconstruction was more detailed and included the posterior bundle of the OR. Postoperatively, the patient had a lower quadrant anopia. At 3 months after the operation, T1WI revealed that a small part of the sagittal stratum had been removed. The area corresponding to the posterior bundle of the OR, responsible for the contralateral lower quadrant field, coincided with the part of the surgically excised area.

\section{DISCUSSION}

The reliability of tractography algorithms as a tool for patient assessment is dependent on factors such as anatomic deviation and compression, intracellular and extracellular edema, tissue infiltration by tumors, fiber degeneration, and inflammation. ${ }^{20}$ Our motivation to include patients with drug-resistant structural epi- 
lepsy being planned for lesion resection includes 2 important points. First, epileptogenic lesions are often circumscribed and are the result of either benign pathologic conditions or low-grade tumors without gross mass effect and changes of the microstructure. Thus, the influence of pathologic microstructure on the performance of the fiber tractography algorithms is thought to be negligible. Second, the postoperative resection area represents an accurately defined lesion in the vicinity of the depicted fiber bundle. Therefore, a verification of the fiber course can be derived in conjunction with the postoperative functional outcome. In 2 patients (patients 14 and 15), this approach could be successfully applied. Some patients were not operated on, because the depicted anatomic course of the fiber tracts was adjacent to the pathologic condition.

The global tracking algorithm successfully reconstructed the DMP arising from the entire convexity of the precentral gyrus. A wider fan angle for the DMP indicates that the crossing with the superior longitudinal fascicle (SLF) and the callosal fiber has been resolved. In particular, the reconstruction of the corticobulbar fibers was established exclusively by this method. They originate in the lateral precentral gyrus, cross the SLF, and terminate in the nuclear areas of the dorsal pons. The large crossing area with the SLF is crucial for tractography algorithms. As expected from the phantom studies, the global tracking efficiently overcame this crossing area. ${ }^{11}$ The success of global tracking lies in its design. Hundreds of segments per voxel represent even those fiber orientations with smaller diffusion weight. The individual line elements are longer than the voxel size, and most importantly, a global model has to be fulfilled in which the reconstructed fibers are hindered to end in the white matter. Thus, the entire fiber system is forced into a configuration where crossings are needed to explain the whole diffusion anisotropy. The reconstruction of the corticobulbar fibers by the other 2 algorithms was inadequate (Fig 1 and On-Line Fig 2). It has been established already that the deterministic FACT cannot solve the problem of fiber crossings with the result of stop of propagation. ${ }^{5}$ Probabilistic algorithms can only partially solve this problem such that the true course of the investigated pathway remains uncertain (supplementary material in ${ }^{11}$ and $^{21}$ ). Postoperatively, no functional deficits were present and no further conclusions could be drawn for the DMP.

For reconstruction of the OR, the probabilistic algorithm performed best. Until now, probabilistic approaches were able to reconstruct its full extent only with an additional manual placement of an ROI anterolateral to the lateral geniculate nucleus. ${ }^{22}$ The probabilistic maps of connectivity used in this study reconstructed the entire extent of the Meyer loop in 9 of 10 patients solely by using the parcellation/atlas-derived ROIs. The distance between the temporal pole tip and the anterior limit of the Meyer loop was in accordance with previous findings ${ }^{23}$ and was an indicator for the detection of the Meyer loop. It has been commonly accepted that the FACT algorithm generally underestimates the Meyer loop. ${ }^{6}$ To exclude effects of the FA threshold of 0.1 , the FACT algorithm was also assessed without it, and this trend did not result in a more reliable detection of the Meyer loop. Global tracking also failed to reconstruct the Meyer loop. This finding is attributed to the rigid alignment of the line elements hindering reconstruction of a sharp bending. A correlation between postoperative visual field defects and the extent of Meyer loop excision has been described before. ${ }^{22}$ In $68 \%-100 \%$ of patients with amygdalohippocampectomy, visual field defects were observed. ${ }^{23}$ One patient subjected to selective amygdalohippocampectomy (patient 14) showed a small upper visual field defect because the Meyer loop was partially damaged. A postoperative lesion was manifest where, in probabilistic maps of connectivity, the most anterior extent of the Meyer loop had been seen correctly. FACT and global tracking showed false-negative results.

In 8 of 10 patients, the estimation of the Meyer loop was not the main aim of tractography because the pathologic condition was located in the occipital lobe or the occipital-temporal region. From this group of patients, 6 were operated on, and a lower quadrant anopia developed in one of these patients (patient 15) postoperatively. In this patient, with a perinatal infarction, the comparison of the postoperative resection area and the preoperatively depicted fibers provided information about the true course of the posterior bundle of the OR. Probabilistic maps of connectivity and global tracking depicted correctly this part of the OR, whereas FACT showed false-negative results for the posterior bundle (Fig 3). Without the FA threshold, the results of FACT were also disappointing. It can be argued that FACT is susceptible to changes of axial and radial diffusivity, which has been recently shown in patients presenting with glioma. ${ }^{21}$ In patient 15 , the course of the OR wound through a postischemic gliotic area visible on T2- and FLAIR-weighted sequences in the sagittal stratum along the ventricle. This finding comprised the whole OR but was most prominent in the posterior bundle.

Our study had several limitations. From a theoretic point of view, it may not have been advisable to compare the simplistic FACT algorithm with the 2 more sophisticated methods. ${ }^{11}$ However, the approach to this study was practical, as it used in vivo data where additional effects of the tissue environment such as anatomic field inhomogeneities may overlay more subtle effects. It was difficult to define similar limiting conditions for all 3 methods. In global tracking, an internal factor, which reflects the brainaveraged anisotropic signal component, serves as an FA threshold. It was chosen such that spurious fibers appear only within areas of FA $<0.1$. For comparability, an FA threshold of 0.1 was applied to the other 2 methods. Another clear limitation of this study was the relatively low number of patients because patients with high-grade tumors were not included in the study. Mass effect and low perifocal FA in high-grade tumors ${ }^{4}$ and changes of diffusion metrics in anaplastic gliomas ${ }^{21}$ have been described as confounding factors for fiber tractography. Therefore, the focus of our study was on well-circumscribed pathologic conditions in patients without mass effect and without gross changes of the microstructure. The validation of tractography approaches still remains a critical issue to be solved. Transcranial magnetic stimulation was contraindicated because of the epilepsy. Intraoperative subcortical mapping is predominantly used in sleep-awake patients $^{24}$ and has not been applied in our institution. Therefore, the course of major fiber bundles could be validated only by postoperative functional deficits. Despite the above-mentioned limitations, the comparison of these methods in a clinical setting is 
new, especially because it considers a global method, which has not been clinically proven so far.

\section{CONCLUSIONS}

The choice of a suitable tractographic algorithm should be a trade-off between the fiber tract of interest and the available computational time. FACT might be sufficient if a fast tractography is required. However, for the Meyer loop of the OR and fiber bundles with strong bending characteristics, probabilistic tractography should be the method of choice. The probabilistic method seems to be the best balance between computational time and effectiveness, and it might be the best choice in most clinical cases. Where a detailed fiber depiction and a resolution of fiber crossings are intended, global tracking should be applied even if it comes along with a prolonged computation time.

Disclosures: Valerij Kiselev—RELATED: Grant: German Research Council,* Comments: Research Grant KI1089/3 - one for development of fiber tracking. Irina Mader-RELATED: Grant: German Research Council, ${ }^{*}$ Comments: German Research Council, MA 2343/4-1; Comprehensive Cancer Centre Freiburg, Germany, Seeding Grant 1027013110. *Money paid to institution

\section{ACKNOWLEDGMENTS}

The authors are grateful to Mr. Hans-Joerg Mast for the MR imaging acquisitions and to Ms. Sophie Aslan for valuable philological suggestions.

\section{REFERENCES}

1. Yogarajah M, Duncan JS. Diffusion-based magnetic resonance imaging and tractography in epilepsy. Epilepsia 2008;49:189-200

2. Basser PJ. Inferring microstructural features and the physiological state of tissues from diffusion-weighted images. NMR Biomed 1995;8:333-44

3. Mori S, Crain BJ, Chacko VP, et al. Three-dimensional tracking of axonal projections in the brain by magnetic resonance imaging. Ann Neurol 1999;45:265-69

4. Nimsky C, Ganslandt O, Hastreiter P, et al. Preoperative and intraoperative diffusion tensor imaging-based fiber tracking in glioma surgery. Neurosurgery 2005;56:130-37; discussion 138

5. Anderson AW. Theoretical analysis of the effects of noise on diffusion tensor imaging. Magn Reson Med 2001;46:1174-88

6. Yamamoto T, Yamada K, Nishimura T, et al. Tractography to depict three layers of visual field trajectories to the calcarine gyri. Am J Ophthalmol 2005;140:781-85

7. Parker GJ, Haroon HA, Wheeler-Kingshott CA. A framework for a streamline-based probabilistic index of connectivity (PICo) using a structural interpretation of MRI diffusion measurements. J Magn Reson Imaging 2003;18:242-54
8. Kreher BW, Schnell S, Mader I, et al. Connecting and merging fibres: pathway extraction by combining probability maps. Neuroimage 2008;43:81-89

9. Kreher BW, Mader I, Kiselev VG. Gibbs tracking: a novel approach for the reconstruction of neuronal pathways. Magn Reson Med 2008;60:953-63

10. Reisert M, Mader I, Anastasopoulos C, et al. Global fiber reconstruction becomes practical. Neuroimage 2011;54:955-62

11. Fillard P, Descoteaux M, Goh A, et al. Quantitative evaluation of 10 tractography algorithms on a realistic diffusion MR phantom. $\mathrm{Neu}$ roimage 2011;56:220-34

12. Behrens TE, Woolrich MW, Jenkinson M, et al. Characterization and propagation of uncertainty in diffusion-weighted MR imaging. Magn Reson Med 2003;50:1077-88

13. Engel J, Van Ness PC, Rasmussen TB, et al. Outcome With Respect to Epileptic Seizures. New York: Raven Press;1993:609-21

14. Wieser HG, Blume WT, Fish D, et al. ILAE Commission Report. Proposal for a new classification of outcome with respect to epileptic seizures following epilepsy surgery. Epilepsia 2001;42:282-86

15. Aganj I, Lenglet C, Sapiro G, et al. Reconstruction of the orientation distribution function in single- and multiple-shell Q-ball imaging within constant solid angle. Magn Reson Med 2010;64:554-66

16. Destrieux C, Fischl B, Dale A, et al. Automatic parcellation of human cortical gyri and sulci using standard anatomical nomenclature. Neuroimage 2010;53:1-15

17. Maldjian JA, Laurienti PJ, Kraft RA, et al. An automated method for neuroanatomic and cytoarchitectonic atlas-based interrogation of fMRI data sets. Neuroimage 2003;19:1233-39

18. Andrews TJ, Halpern SD, Purves D. Correlated size variations in human visual cortex, lateral geniculate nucleus, and optic tract. J Neurosci 1997; 17:2859-68

19. Fillard P, Poupon C, Mangin JF. A novel global tractography algorithm based on an adaptive spin glass model. Med Image Comput Comput Assist Interv 2009;12:927-34

20. Alexander AL, Lee JE, Lazar M, et al. Diffusion tensor imaging of the brain. Neurotherapeutics 2007;4:316-29

21. Nguyen-Thanh T, Reisert M, Anastasopoulos C, et al. Global tracking in human gliomas: a comparison with established tracking methods. Clin Neuroradiol 2013;23:263-75

22. Yogarajah M, Focke NK, Bonelli S, et al. Defining Meyer's looptemporal lobe resections, visual field deficits and diffusion tensor tractography. Brain 2009;132:1656-68

23. Mandelstam SA. Challenges of the anatomy and diffusion tensor tractography of the Meyer loop. AJNR Am J Neuroradiol 2012;33: 1204-10

24. Bello L, Gambini A, Castellano A, et al. Motor and language DTI fiber tracking combined with intraoperative subcortical mapping for surgical removal of gliomas. Neuroimage 2008;39:369-82

25. Palmini A, Najm I, Avanzini G, et al. Terminology and classification of the cortical dysplasias. Neurology 2004;62:S2-8

26. Ebeling U, Reulen HJ. Neurosurgical topography of the optic radiation in the temporal lobe. Acta Neurochir (Wien) 1988;92:29-36 